\title{
Rho/Rho-associated protein kinase signaling pathway-mediated downregulation of runt-related transcription factor 2 expression promotes the differentiation of dental pulp stem cells into odontoblasts
}

\author{
XIAOQING HUANG ${ }^{1,2}$, XIAOLING CHEN ${ }^{2}$, HONGBAI CHEN ${ }^{3}$, DONGWEI XU ${ }^{2}$, CHEN LIN $^{2}$ and BIN PENG ${ }^{4}$ \\ ${ }^{1}$ Department of Endodontics, School and Hospital of Stomatology, Wuhan University, Wuhan, Hubei 430079; Departments \\ of ${ }^{2}$ Endodontics and ${ }^{3}$ Periodontics, Xiamen Stomatological Hospital, Xiamen, Fujian 361003; ${ }^{4}$ State Key Laboratory, \\ Breeding Base of Basic Science of Stomatology and Key Laboratory of Oral Biomedicine Ministry of Education, \\ School and Hospital of Stomatology, Wuhan University, Wuhan, Hubei 430079, P.R. China
}

Received September 28, 2016; Accepted July 20, 2017

DOI: $10.3892 /$ etm.2018.5982

\begin{abstract}
The present study investigated the role of runt-related transcription factor 2 (Runx2) in regulating the differentiation of human dental pulp stem cells (hDPSCs) into odontoblasts under the mediation of the Rho/Rho-associated protein kinase (ROCK) signaling pathway. hDPSCs and human bone marrow mesenchymal stem cells (hBMSCs) were mineralized to induce differentiation. The expression levels of odontoblast- and osteoblast-specific proteins, dentin sialophosphoprotein (DSPP), osteocalcin (OCN) and Runx2, were measured using western blot analysis. The hDPSCs were treated with Rho/ROCK signaling pathway inhibitor, $\mathrm{C} 3$ exoenzyme, and mineralized prior to determining the protein expression levels of RhoA, ROCK, Runx2, OCN, DSPP, and mRNA expression levels of early mineralization genes, including alkaline phosphatase, collagen type I, Msh homeobox 2 and distal-less homeobox 2, and late mineralization genes, including DSPP, dentin matrix protein-1 (DMP-1), bone sialoprotein (BSP) and $O C N$. Flow cytometry data indicated that $95 \%$ of the isolated hDPSCs were positive for mesenchymal stem cell markers, including cluster of differentiation (CD)29, CD90 or CD105, and vascular endothelial cell marker, CD146, whereas $<5 \%$ of the hDPSCs were positive for hematopoietic stem cell markers, CD34 and CD45. The
\end{abstract}

Correspondence to: Professor Bin Peng, State Key Laboratory, Breeding Base of Basic Science of Stomatology and Key Laboratory of Oral Biomedicine Ministry of Education, School and Hospital of Stomatology, Wuhan University, 237 Luoyu Road, Wuhan, Hubei 430079, P.R. China

E-mail: phs301@whu.edu.cn

Key words: Rho/Rho-associated protein kinase signaling pathway, runt-related transcription factor 2 , human dental pulp stem cells, odontoblast differentiation expression levels of DSPP in hDPSCs and OCN in hBMSCs were significantly upregulated with increased time in mineralization medium $(\mathrm{P}<0.01)$, which suggested that hDPSCs and hBMSCs were differentiated into odontoblasts and osteoblasts, respectively. During the osteogenic process, Runx 2 protein was highly expressed in mesenchymal stem cells following stimulation with mineralization medium compared with cells that received no stimulation. During odontoblast differentiation in hDPSCs, Runx2 protein was highly expressed in the early stage; however, the expression declined in the late stage. Furthermore, treatment with C3 exoenzyme significantly downregulated the expression of RhoA, ROCK and Runx2 compared with the control in hDPSCs $(\mathrm{P}<0.01)$. Additionally, in mineralization solution, $\mathrm{C} 3$ exoenzyme also significantly downregulated the expression of Runx2 $(\mathrm{P}<0.01)$; however, the Rho/ROCK signaling pathway inhibitor did not significantly impact the expression of early mineralization genes. By contrast, C3 exoenzyme significantly upregulated the expression of DSPP and DMP-1, and downregulated the expression of BSP and OCN $(\mathrm{P}<0.01)$. The present findings suggested that odontoblast differentiation in hDPSCs may be regulated by Rho/ROCK signaling pathway-mediated downregulation of Runx2.

\section{Introduction}

Genetic diseases, trauma and caries may lead to tooth loss. Implants or dentures are used clinically to repair missing teeth $(1,2)$. However, there are a number of disadvantages in these approaches (3). For example, the implanted or denture tooth is generally not as strong as the natural tooth (3). With developments in tissue engineering, it is becoming possible to use stem cells and tissue engineering techniques to repair defected teeth $(1,2)$. Dental pulp stem cells (DPSCs) are mesenchymal stem cells (MSCs) capable of differentiating into neural cells, adipocytes and odontoblasts (4). Studies have demonstrated that DPSCs may be used to repair the defected tooth; however, how DPSCs are directionally differentiated into 
odontoblasts remains unclear (5-7). Studies have demonstrated that during the differentiation of DPSCs into odontoblasts the undifferentiated cells are smaller than the differentiated cells (4). DPSCs have been demonstrated to differentiate into a variety of cell types. Depending on induction conditions DPSCs may differentiate into odontoblasts, myotubes or adipocytes $(8,9)$. hDPSCs isolated from dental pulp of young permanent teeth may differentiate into osteoblasts in vitro (10). In addition, DPSCs also express markers for glial cells and neuronal precursors, including glial fibrillary acidic proteins and nestin, and may differentiate into neurons in vitro; indicating that cultured DPSCs have the potential to differentiate into neuron-like cells (11-13). Studies have also revealed that DPSCs can differentiate into chondrocytes in vitro $(13,14)$.

Runt-related transcription factor 2 (Runx2), also known as core binding factor $\alpha 1$, belongs the Runt domain gene family of transcription factors. It regulates protein expression at transcriptional level and serves a crucial role in bone and tooth development (15). A previous study demonstrated that, as an osteoblast differentiation-specific transcription factor, Runx2 may directly induce the differentiation of mesenchymal stem cells to osteoblasts (16). Runx 2 is expressed in odontogenic and osteogenic mesenchymal cells during the embryonic period, and its expression patterns in osteoblast differentiation and odontoblast differentiation are different (17-19). Therefore, it may be a key attribute that determines odontoblast differentiation.

The Rho/Rho-associated protein kinase (ROCK) signaling pathway has been indicated to have a role in cell proliferation, differentiation and apoptosis, and in regulating the differentiation of MSCs into osteoblasts and adipocytes (20). Therefore, the Rho/ROCK signaling pathway may also regulate the expression of Runx2 to direct the differentiation of human dental pulp stem cells (hDPSCs) into odontoblasts (21). The aim of the present study was to investigate the expression of Runx 2 during odontoblast and osteoblast differentiation and effect of the Rho/ROCK signaling pathway on the expression of Runx 2 and other mineralization genes using a pathway inhibitor during odontoblast differentiation.

\section{Materials and methods}

Human bone marrow MSCs (hBMSCs). hBMSCs were purchased from the American Type Culture Collection (Manassas, VA, USA) and maintained in Dulbecco's modified Eagle's medium (DMEM) supplemented with $12 \%$ fetal bovine serum (FBS; Gibco; Thermo Fisher Scientific, Inc., Waltham, $\mathrm{MA}, \mathrm{USA}$ ) at $37^{\circ} \mathrm{C}$ in a humidified atmosphere containing $5 \%$ $\mathrm{CO}_{2}$. The present study was approved by the Ethics Committee of Xiamen Stomatological Hospital (Xiamen, China).

Reagents and instruments. A BCA protein assay kit, enhanced chemiluminescent ultra-sensitive luminescence solution, mouse anti-GAPDH monoclonal antibodies (cat. no. AF0006) and horseradish peroxidase-labeled goat anti-rabbit immunoglobulin $\mathrm{G}(\mathrm{H}+\mathrm{L})$ antibodies (cat. no. A0208) were purchased from Beyotime Institute of Biotechnology (Beijing, China). Peroxidase-conjugated goat anti-mouse $\operatorname{IgG}(\mathrm{H}+\mathrm{L})$ antibodies (cat. no. ZB-2305) were purchase from OriGene Technologies, Inc. (Rockville, MD, USA). Rabbit anti-dentin sialophosphoprotein (DSPP; cat. no. ab216892), osteocalcin (OCN; cat. no. ab13420), Runx2 (cat. no. ab76956), RhoA (cat. no. ab54835) and ROCK (cat. no. ab45171) polyclonal antibodies, anti-cluster of differentiation (CD)29-phycoerythrin (PE) (cat. no. ab93759), CD90-PE (cat. no. ab93758), CD105-PE (cat. no. ab11414), CD146-PE (cat. no. ab75769), CD34-PE (cat. no. ab81289) and CD45-PE (cat. no. ab10558) antibodies were purchased from were purchased from Abcam (Cambridge, MA, USA). Hank's balanced salt solution (HBSS) and radioimmunoprecipitation assay (RIPA) lysis buffer were from Shanghai Solarbio Science \& Technology Co., Ltd. (Shanghai, China). Collagenase I and neutral collagenase II were obtained from Gibco (Thermo Fisher Scientific, Inc.) and a TRIzol kit and one-step reverse transcription (RT)-polymerase chain reaction (PCR) kit were obtained from Invitrogen (HiFiScript cDNA synthesis kit; cat no. CW2569M; CWBIO, Beijing, China). C3 exoenzyme was purchased from Sigma-Aldrich (Merck KGaA, Darmstadt, Germany). The flow cytometer FACScalibur was supplied by BD Biosciences (San Jose, CA, USA). Evolution ${ }^{\mathrm{TM}} 220 \mathrm{UV}^{\mathrm{U}} \mathrm{Visible}$ spectrophotometer was from Thermo Fisher Scientific, Inc. and electrophoresis apparatus, electrophoresis transfer apparatus and the gel imaging system (ChemiDocTM XRS) were obtained from Bio-Rad Laboratories, Inc. (Hercules, CA, USA). The fluorescence quantitative PCR instrument LightCycler96 was purchased from Roche Diagnostics (Basel, Switzerland) and the PCR thermal cycler PTC-200 was purchased from (Bio-Rad Laboratories, Inc.).

hDPSC isolation. hDPSCs were isolated as described previously $(22,23)$. Impacted third molars were collected from 29 healthy young (aged 19-29) male volunteers; the pulps were extracted under sterile conditions. The volunteers who possessed healthy teeth tissue, and no periodontal or periapical diseases were enrolled in the present study at Xiamen Stomatological Hospital (Xiamen, China) between July and December 2015. Patients who had previously had extraction were excluded. All volunteers provided their prior written, informed consent. The pulps were washed with HBSS, and digested with $3 \%$ collagenase I and $4 \%$ neutral collagenase II for $1 \mathrm{~h}$ at $37^{\circ} \mathrm{C}$, and filtered through a $70 \mu \mathrm{m}$ mesh to generate a single cell suspension. The suspension was centrifuged at $500 \mathrm{x} \mathrm{g}$ at $25^{\circ} \mathrm{C}$ for $10 \mathrm{~min}$, resuspended in DMEM supplemented $15 \%$ FBS, inoculated into wells in 96-well plates and cultured at $37^{\circ} \mathrm{C}$ in an atmosphere containing $5 \% \mathrm{CO}_{2}$. The culture medium was refreshed every 3 days and subcultured when the confluency reached $80-90 \%$.

hDPSC characterization. hDPSCs at the third passage were digested with $0.25 \%$ EDTA-trypsin at $37^{\circ} \mathrm{C}$ for 2 min when the confluency reached $80-90 \%$ to obtain a single cell suspension. The cells were adjusted to a density of $1 \times 10^{6}$ cells $/ \mathrm{ml}$ and incubated with CD29-PE, CD90-PE, CD105-PE, CD146-PE, CD34-PE and CD45-PE antibodies (all 1:100) for $1 \mathrm{~h}$ at room temperature and analyzed by performing flow cytometry using a flow cytometer. The cells were blocked in $1 \%$ bovine serum albumin (BSA; CWBIO) for $30 \mathrm{~min}$ at $37^{\circ} \mathrm{C}$ and washed with PBS prior to the analysis. The data were analysed using BD CellQuest ${ }^{\mathrm{TM}}$ software (version 5.1; BD Biosciences). 
Table I. Reverse transcription-quantitative polymerase chain reaction primers.

\begin{tabular}{|c|c|c|c|}
\hline Gene of interest & Direction & Primer sequence $\left(5^{\prime}-3^{\prime}\right)$ & Expected product size (bp) \\
\hline \multirow[t]{2}{*}{ Alkaline phosphatase } & Forward & CTCGTTGACCACCTGGAAGAGCTTCAAACCG & 168 \\
\hline & Reverse & GGTCCGTCACGTTGTTCCTGTTCAGC & \\
\hline \multirow[t]{2}{*}{ Collagen type I } & Forward & AGGGCTCCAACGAGATCGAGATCCG & 223 \\
\hline & Reverse & TACAGGAAGCAGACAGGGCCAACGTCG & \\
\hline \multirow[t]{2}{*}{ Msh homeobox 2} & Forward & CATCCCAGCTTCTAGCCTTC & 103 \\
\hline & Reverse & GCAGCCATTTTCAGCTTTTC & \\
\hline \multirow[t]{2}{*}{ Distal-less homeobox 2} & Forward & GGCTCCTACCAGTCCCACG & 133 \\
\hline & Reverse & CGTTGTTGACCGGAGACGAA & \\
\hline \multirow[t]{2}{*}{ Dentin sialophosphoprotein } & Forward & GTACCCAAACAACACAGGACA & 114 \\
\hline & Reverse & AATCAGGGATTAGAAACCGAAG & \\
\hline \multirow[t]{2}{*}{ Dentin matrix protein-1 } & Forward & CATGACACCTTTGGAGACGAA & 145 \\
\hline & Reverse & CTTGGGCACTGTTTTCTTGAG & \\
\hline \multirow[t]{2}{*}{ Bone sialoprotein } & Forward & GCAGTTAGCAATACTACAGGCA & 111 \\
\hline & Reverse & GACCATTTATCAAGCAGTACACC & \\
\hline \multirow[t]{2}{*}{ Osteocalcin } & Forward & CATGAGAGCCCTCACA & 315 \\
\hline & Reverse & AGAGCGACACCCTAGAC & \\
\hline \multirow[t]{2}{*}{ GAPDH } & Forward & AGCCACATCGCTCAGACA & 314 \\
\hline & Reverse & TGGACTCCACGACGTACT & \\
\hline
\end{tabular}

Induction and characterization of odontoblasts and osteoblasts. hDPSCs and hBMSCs at the third passage were inoculated to the wells of 6-well plates, combined with $100 \mu 1$ mineralization induction solution $(10 \mathrm{nmol} / 1$ dexamethasone, $10 \mathrm{mmol} / \mathrm{l} \beta$-glycerophosphate and $50 \mu \mathrm{g} / \mathrm{ml}$ vitamin $\mathrm{C}$ ) and $\alpha$-minimum essential medium (Gibco; Thermo Fisher Scientific, Inc.) supplemented with $100 \mathrm{ml} / 1 \mathrm{FBS}$, and cultured at $37^{\circ} \mathrm{C}$. Cells were harvested at day $0,7,14$ and 21 for analysis of DSPP, OCN and Runx2 levels using western blot analysis.

Treatment with $C 3$ exoenzyme. hDPSCs $\left(1 \times 10^{6}\right)$ at the third passage were inoculated into the wells of 6 -well plates and $\mathrm{C} 3$ exoenzyme at a final concentration of $50 \mu \mathrm{g} / \mathrm{ml}$ was added. Following $48 \mathrm{~h}$ of incubation at $37^{\circ} \mathrm{C}$, cells were harvested and assessed for the expression of RhoA, ROCK, Runx2, alkaline phosphatase (ALP), collagen type I (Col I), Msh homeobox 2 (MSX2), distal-less homeobox 2 (DLX2), DSPP, dentin matrix protein-1 (DMP-1), bone sialoprotein (BSP) and OCN at the protein or mRNA level. Cells cultured in DMEM and not treated with $\mathrm{C} 3$ exoenzyme were used as control.

RT-quantitative PCR (RT-qPCR). Total RNA was extracted hDPSCs and hBMSCs using a TRIzol kit, according to the manufacturer's protocols. The purity and quantity of RNA were determined using a UV spectrophotometer. The RT reaction was performed with $200 \mathrm{ng}$ RNA in a total volume of $10 \mu \mathrm{l}$ containing $2 \mu \mathrm{l}(2 \mu \mathrm{g})$ RNA, $2 \mu \mathrm{l} \mathrm{dNTP}(500 \mu \mathrm{g} / \mathrm{ml})$ and $2 \mu \mathrm{MgCl}_{2}(50 \mathrm{mM})$, and the resulting complementary DNA was quantified using a one-step PCR kit, according to the manufacturer's protocols, and the primers listed in Table I in a total volume of $25 \mu$ l. Human GADPH (Hs03929097_g1), was used as an internal control. The cycling conditions performed were the following: Pre-denaturation at $95^{\circ} \mathrm{C}$ for
$5 \mathrm{~min}$, followed by 40 cycles of $30 \mathrm{sec}$ at $95^{\circ} \mathrm{C}, 30 \mathrm{sec}$ at $60^{\circ} \mathrm{C}$ and $30 \mathrm{sec}$ at $72^{\circ} \mathrm{C}$. qPCR was performed using 30 cycles of denaturation at $95^{\circ} \mathrm{C}$ for $30 \mathrm{sec}$, annealing at $51^{\circ} \mathrm{C}$ for $20 \mathrm{sec}$ and extension at $75^{\circ} \mathrm{C}$ for $30 \mathrm{sec}$. Experiments were performed in triplicate and the mean value was calculated for each case. The data were assessed using RQ Manager software (version 1.2.1.; Applied Biosystems; Thermo Fisher Scientific, Inc.). Relative expression was calculated by using the $2^{-\Delta \Delta \mathrm{Cq}}$ method and obtaining the fold change value, according to previously described protocol (17).

Western blot analysis. Following different treatments, hDPSCs and hBMSCs were lysed with RIPA lysis buffer that contained protease and phosphatase inhibitors. The supernatants were collected following centrifugation at 7,000 $\mathrm{x} \mathrm{g}$ at $4^{\circ} \mathrm{C}$ for $20 \mathrm{~min}$. The protein was quantified using the BCA method, $20 \mu \mathrm{g}$ protein was loaded per lane and separated using 12\% SDS-PAGE, transferred to a PVDF membrane. The membranes were blocked with $2 \%$ BSA (Beyotime Institute of Biotechnology) at room temperature for $1 \mathrm{~h}$, then the target proteins were detected by incubating the membrane with primary antibodies against DSPP, osteocalcin, Runx2, RhoA, ROCK and GAPDH (all 1:100) at $4^{\circ} \mathrm{C}$ overnight, and horseradish peroxidase-labeled goat anti-rabbit secondary and peroxidase-conjugated goat anti-mouse antibodies (both $1: 2,000$ ) at $37^{\circ} \mathrm{C}$ for $1 \mathrm{~h}$. Visualization was achieved with a chemiluminescence kit. The intensity of blot signals was quantitated using Quantity one analysis software (version 4.62; Bio-Rad Laboratories, Inc.).

Statistical analysis. All data are expressed as the mean \pm standard error of the mean obtained from at least three independent experiments. Statistical comparisons between 
groups were assessed by using the Student's t-test. $\mathrm{P}<0.05$ was considered to indicate a statistically significant difference. Data were analyzed using SPSS software (version 10.1; SPSS, Inc., Chicago, IL, USA).

\section{Results}

hDPSCs are spindle-shaped, and positive for MSC and endothelial cell markers. As indicated in Fig. 1, the primary hDPSCs were spindle-shaped and fibrous-like after 3 days of culture in vitro. The culture reached $80 \%$ confluence in 7-8 days. As indicated in Fig. 2, flow cytometry analysis revealed that $>95 \%$ of the cells were positive for MSC markers CD29, CD90, CD105, and for endothelial cell marker CD146, whereas $<5 \%$ were positive for hematopoietic stem cell markers CD34 and CD45. These results suggested that the purity of the hDPSCs was high enough for the subsequent experiments.

DSPP protein expression increases throughout the differentiation of hDPSCs into odontoblasts. The differentiation of hDPSCs into odontoblast was investigated by determining the protein expression levels of DSPP. Results indicated that, following treatment with mineralization induction solution, the protein expression levels of DSPP in hDPSCs were increased in a time-dependent manner, and at 7,14 and 21 days, expression levels were significantly increased compared with 0 days $(\mathrm{P}<0.01$; Fig. 3). These findings suggested that odontoblast differentiation may have been induced in the hDPSCs.

OCN protein expression increases throughout the differentiation of hBMSCs into osteoblast. The protein expression levels of OCN in hBMSCs cultured in mineralization solution were also examined in the present study. The data demonstrated that the protein expression levels of OCN were significantly upregulated at 14 and 21 days after induction compared with 0 days $(\mathrm{P}<0.01$; Fig. 4$)$, which indicated that mineralization may have induced hBMSCs to differentiate into osteoblasts.

Runx2 protein expression decreases in hDPSCs and increases in hBMSCs during odontoblast and osteoblast differentiation, respectively. In hDPSCs, increased incubation time in mineralization medium resulted in increased protein expression levels of Runx 2 until day 5, after which the expression levels declined. Furthermore, significantly increased expression levels of Runx 2 protein were indicated at 3, 5, 7 and 14 days in culture compared with 0 days in culture $(\mathrm{P}<0.01$; Fig. 5A). Conversely, the expression of Runx 2 protein in hBMSCs was increased in a time-dependent manner, with a significant increase in expression indicated at $5(\mathrm{P}<0.05), 7(\mathrm{P}<0.01), 14(\mathrm{P}<0.01)$ and $21(\mathrm{P}<0.01)$ days compared with 0 days (Fig. 5B).

C3 exoenzyme incubation decreased Runx2, Rho and ROCK protein expression in hDPSCs. To examine whether the Rho/ROCK signaling pathway is involved in regulating Runx 2 expression, hDPSCs were treated with the pathway inhibitor, C3 exoenzyme. Results indicated that compared with the control, hDPSCs treated with the inhibitor exhibited significantly decreased protein expression levels of RhoA, ROCK and Runx2 (P<0.01; Fig. 6A). Furthermore, following

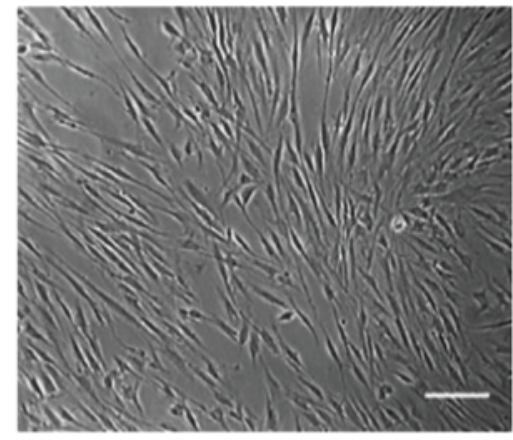

Figure 1. hDPSCs are spindle-shaped. Primary hDPSCs after 3 days of in vitro culture. Magnification, $\mathrm{x} 200$. hDPSCs, human dental pulp stem cells Scale bar, $100 \mu \mathrm{M}$.

mineralization induction, Runx 2 protein expression levels in the inhibitor-treated hDPSCs were significantly decreased at 7 and 14 days compared with that in the control $(\mathrm{P}<0.01$; Fig. 6B).

C3 exoenzyme incubation alters the $m R N A$ expression of late mineralization genes. The present study evaluated the impact of the Rho/ROCK signaling pathway on the mRNA expression levels of early and late mineralization genes. Compared with the control, the mRNA expression levels of early mineralization genes ALP, Col I, MSX2 and DLX2 were not significantly different in inhibitor-treated hDPSCs (Fig. 7A). However, the mRNA expression levels of late mineralization genes, DSPP and DMP-1, were significantly upregulated in inhibitor-treated hDPSCs, whereas BSP and OCN were significantly downregulated compared with the controls $(\mathrm{P}<0.01$; Fig. $7 \mathrm{~B})$.

\section{Discussion}

Research has demonstrated that DPSCs may be isolated and cultured in vitro (24). DPSCs form dentin-like complex structures on the fracture surfaces in the teeth of immunodeficient nude mice or dogs where DPSC-seeded stents were implanted (25), which suggests that, under certain conditions, DPSCs may differentiate to form the pulp-dentin complex and may be used as seed cells for pulp tissue regeneration and dentin repair. To elucidate the molecular regulation of odontoblast differentiation in hDPSCs, the expression of genes involved in the differentiation and impact of the Rho/ROCK signaling pathway were investigated in the present study. To accompany these, hDPSCs were isolated with high purity according to microscopy and flow cytometry analysis. Furthermore, the present results also demonstrated that the isolated hDPSCs may differentiate toward odontoblasts in vitro as the expression of DSPP, an odontoblast-specific protein (19), was significantly increased over time when the hDPSCs were cultured in mineralization induction medium. Additionally, results indicated that hBMSCs may be induced by the mineralization medium to differentiate into osteoblasts, as the protein expression levels of osteoblast-specific protein, OCN, were significantly upregulated following 14 and 21 days in culture compared with 0 days. OCN is vitamin K-dependent small molecule protein, and has high affinity to calcium and hydroxyapatite (26). Therefore, OCN is regarded as late stage marker for bone formation (26). 

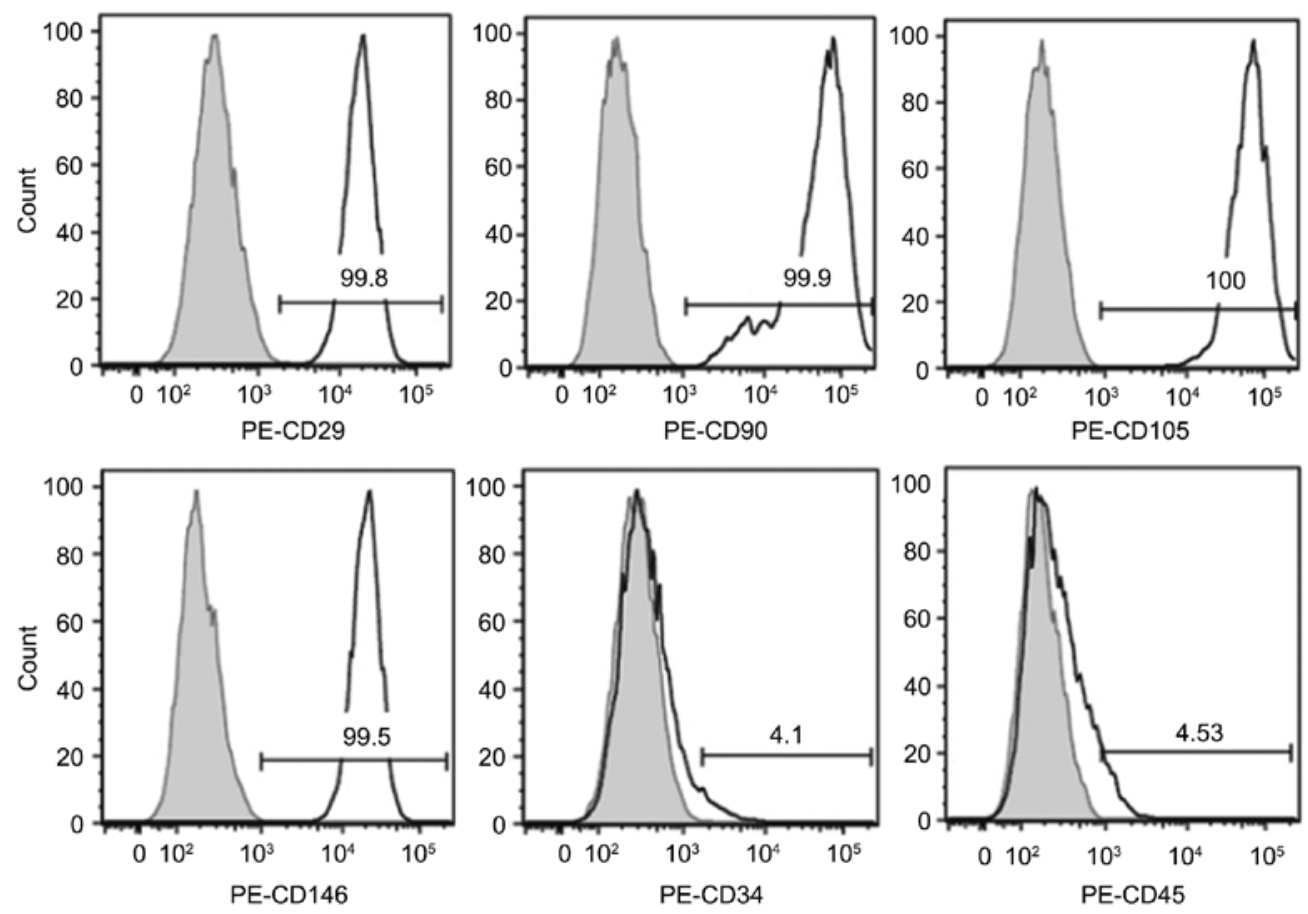

Figure 2. Human dental pulp stem cells are positive for mesenchymal stem cell and endothelial cell markers. Flow cytometry results of cell surface markers, including CD29, CD90, CD105 and CD146, in human dental pulp stem cells. CD, cluster of differentiation; PE, phycoerythrin.

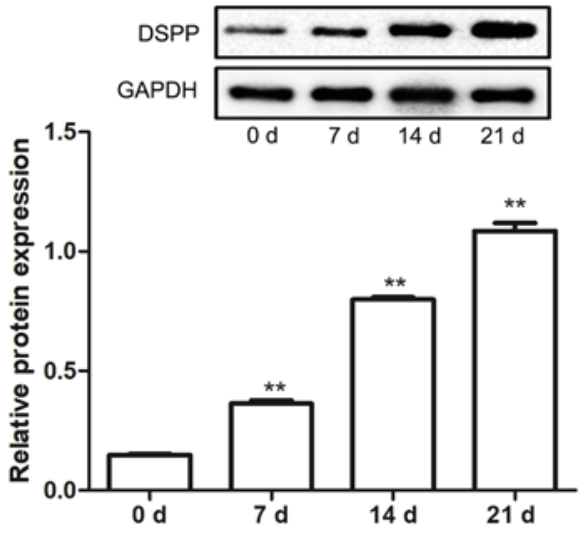

Figure 3. DSPP protein expression increases throughout the differentiation of human dental pulp stem cells into odontoblasts. Protein expression levels of DSPP were determined using western blot analysis. Data are presented as the mean \pm standard error of the mean of at least three experiments. ${ }^{* *} \mathrm{P}<0.01$ vs. 0 d. DSPP, dentin sialophosphoprotein; d, day.

Runx2, a member of the Runt family, is expressed in osteogenic and odontogenic mesenchymal cells during the embryonic period, and has an important role in bone and tooth development $(23,27)$. It is capable of binding specific sites on promoter sequences to regulate the transcription of downstream genes and, subsequently, the development of tissues and organs $(23,27)$. Studies have revealed that Runx2 may regulate the differentiation of BMSCs into osteoblasts, which has an important role in bone tissue repair, and it is continuously and highly expressed during osteogenic differentiation $(17,19)$. During the differentiation of hBMSCs to odontoblasts, Runx 2 expression increases in the early stage and declines in the late stage $(17,19)$, which suggests that the expression pattern of Runx 2 may be a key factor that impacts

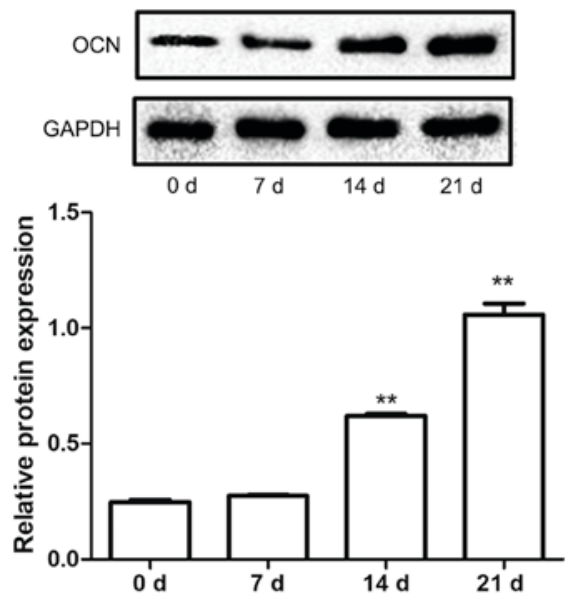

Figure 4. OCN protein expression increases throughout the differentiation of human bone marrow mesenchymal stem cells into osteoblast. Protein expression levels of OCN were determined using western blot analysis. Data are presented as the mean \pm standard error of the mean of at least three experiments. ${ }^{* *} \mathrm{P}<0.01$ vs. 0 d. $\mathrm{OCN}$, osteocalcin; $\mathrm{d}$, day.

the differentiation direction to odontoblast or to osteoblast. The present study indicated that, following mineralization induction, Runx 2 protein expression patterns in hDPSCs and hBMSCs were different. In hDPSCs, Runx 2 protein expression levels increased in the early differentiation period, peaked on day 5 and then declined in late differentiation period. However, in hBMSCs, Runx 2 protein expression levels increased over the full duration of the experimental period and in the late mineralization stage. These results are consistent with earlier studies $(24,25)$, which implies that regulating Runx 2 expression may influence the direction of differentiation in these stem cells. 

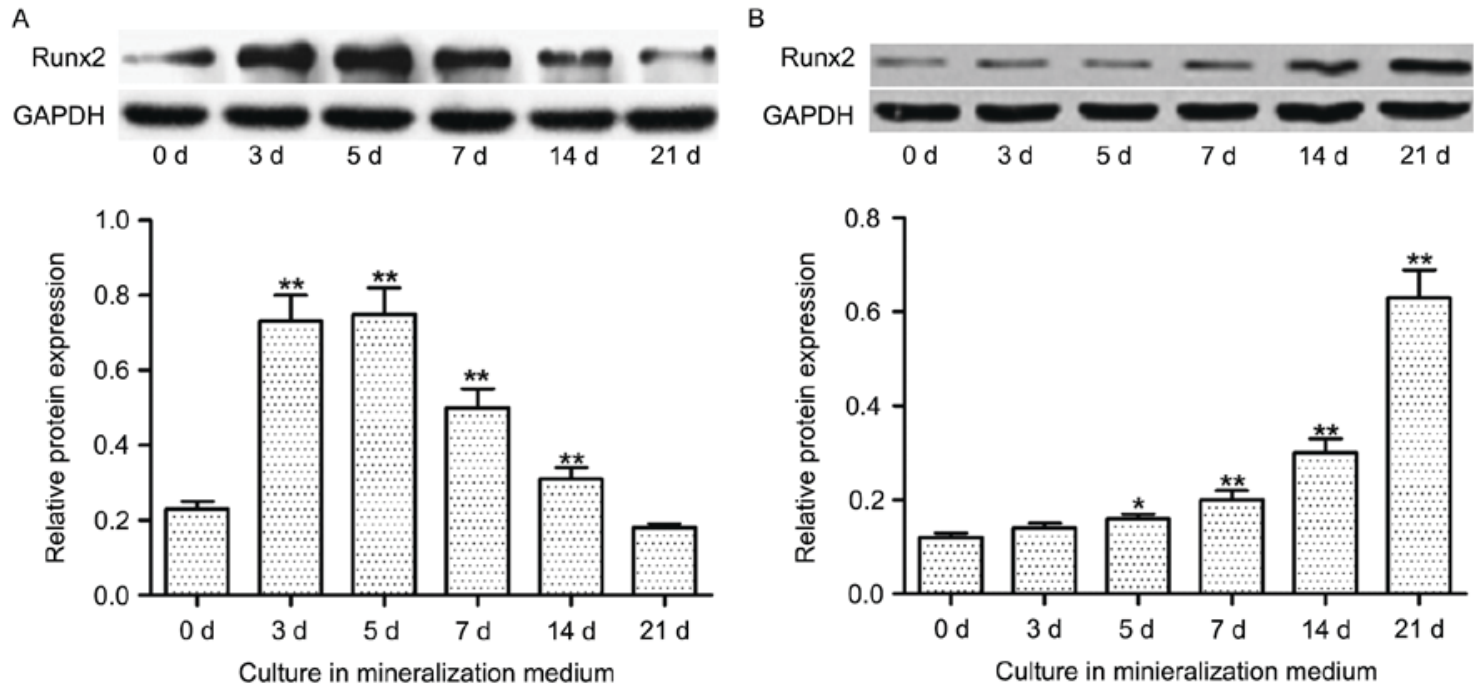

Figure 5. Runx2 protein expression decreases in hDPSCs and increases in hBMSCs during odontoblast and osteoblast differentiation, respectively. The expression of Runx2 in (A) hDPSCs during odontoblast differentiation and (B) hBMSCs during osteoblast differentiation. Cells were incubated in mineralization medium for $0,3,5,7,14$ or 21 days. Protein expression levels of Runx 2 were determined using western blot analysis. Data are presented as the mean \pm standard error of the mean of at least three experiments. ${ }^{*} \mathrm{P}<0.05$ and ${ }^{* *} \mathrm{P}<0.01$ vs. $0 \mathrm{~d}$. Runx2, runt-related transcription factor 2 ; d, day; hDPSCs, human dental pulp stem cells; hBMSCs, human bone marrow mesenchymal stem cells.

A

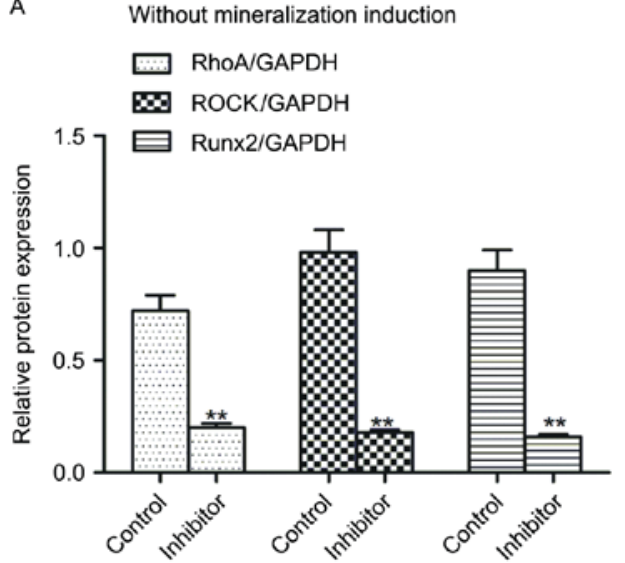

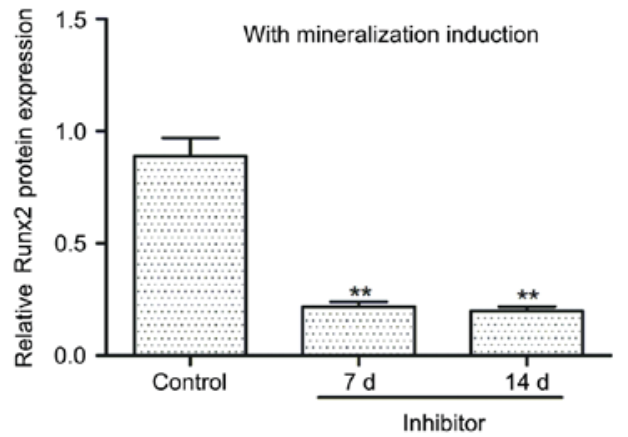

Figure 6. C3 exoenzyme incubation decreased Runx2, Rho and ROCK protein expression in human dental pulp stem cells. Regulation of Runx2, RhoA and ROCK protein expression by the Rho/ROCK signaling pathway in human dental pulp stem cells (A) without and (B) with mineralization treatment. The inhibitor was C3 exoenzyme. Cells that received mineralization treatment were incubated for 0 (control), 7 or 21 days. Data are presented as the mean \pm standard error of the mean of at least three experiments. ${ }^{* *} \mathrm{P}<0.01$ vs. Control. Runx 2 , runt-related transcription factor 2 ; ROCK, Rho-associated protein kinase.
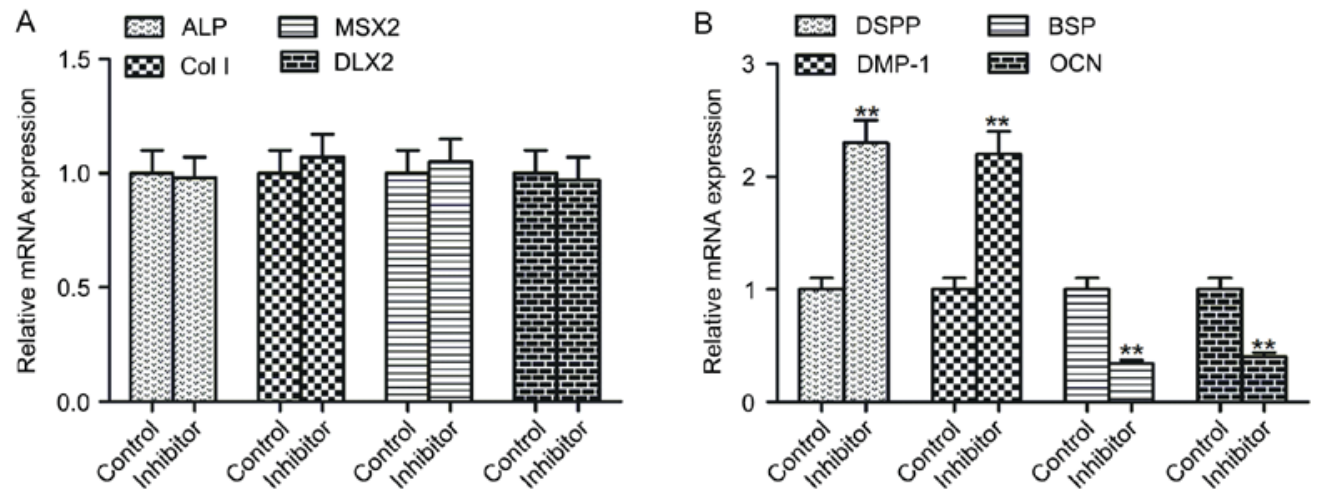

Figure 7. C3 exoenzyme incubation alters the mRNA expression of late mineralization genes. Relative mRNA expression levels of (A) early and (B) late mineralization genes in human dental pulp stem cells without or with treatment using the Rho/Rho-associated protein kinase signaling pathway inhibitor. The inhibitor was $\mathrm{C} 3$ exoenzyme. Data are presented as the mean \pm standard error of the mean of at least three experiments. ${ }^{* *} \mathrm{P}<0.01 \mathrm{vs}$. Control. OCN, osteocalcin; ALP, alkaline phosphatase; Col I, collagen type I; DSPP, dentin sialophosphoprotein; DMP-1, dentin matrix protein-1; BSP, bone sialoprotein; MSX2, Msh homeobox 2; DLX2, distal-less homeobox 2. 
The Rho/ROCK signaling pathway is associated with cell proliferation and differentiation, apoptosis, the cell cycle, cell polarity, the cytoskeleton and vasoconstriction $(23,27,28)$. The signaling pathway also regulates the differentiation of MSCs to adipocytes, osteoblasts and chondrocytes $(11,13)$. The upstream protein Rho GTPase belongs to the Ras superfamily, including RhoA, RhoB and RhoC subfamilies $(28,29)$. Rho GTPase exists in all eukaryotic organisms and has roles in cell migration, movement, proliferation and differentiation $(28,29)$. Rho GTPase has a number of downstream effector proteins, including fatty kinase and serine/threonine kinases (30). Among them, ROCK has been intensively studied. Activated RhoA may bind directly to the C-terminus of ROCK to activate it (31). Activated ROCK phosphorylates myosin and its regulatory proteins to regulate changes of and contract the cytoskeleton (31). Therefore, RhoA/ROCK signaling is important for cytoskeleton reorganization, cell migration, movement, contraction and proliferation (32). Furthermore, the RhoA/ROCK signaling influences cell polarity and morphology by regulating cytoskeletal polymerization, thereby affecting the final differentiation of a cell (32). -Studies have indicated that the regulation of the RhoA/ROCK signaling pathway induces the differentiation of cells toward osteoblasts $(33,34)$. For example, through activating the ROCK signaling pathway, BMSCs were prompted to differentiate into osteoblasts with increased Runx2 and osteocalcin expression observed (35). However, an alternative study indicated that inhibition of the ROCK signaling pathway decreased LIPUS-induced differentiation of MSCs to osteoblasts and downregulated Runx2 expression (36). These studies demonstrate that the RhoA/ROCK signaling pathway may have regulatory roles on Runx 2 expression in osteoblasts and suggests that the pathway may also have a role in regulating Runx 2 expression in odontoblast differentiation. In the present study, the RhoA/ROCK signaling pathway inhibitor, C3 exoenzyme (37), was used to analyze the impact of the signaling pathway in hDPSCs. Results demonstrated that C3 exoenzyme treatment significantly downregulated the protein expression levels of RhoA, ROCK and Runx 2 in hDPSCs. Furthermore, the protein expression levels of Runx2 were also significantly decreased following culture for 7 and 14 days in mineralization medium. These findings suggest that the RhoA/ROCK signaling pathway may have a regulatory role on Runx 2 expression in hDPSCs during dentin differentiation.

To provide further insights on how the RhoA/ROCK signaling pathway regulates the differentiation of odontoblasts, the impact of the pathway on the expression of odontoblast phenotype proteins was explored. Results indicated that the pathway inhibitor did not affect the transcription of early mineralization genes, suggesting that the pathway is not involved in the early stage of odontoblast differentiation. However, inhibition of the pathway led to significant upregulation of DSPP and DMP-1, and significant downregulation of BSP and OCN late mineralization genes. DSPP and DMP-1 are only expressed in odontoblasts and their promoters contain Runx2 binding sites $(38,39)$. The expression of DSPP and DMP-1 has been demonstrated to be regulated by Runx 2 to direct the differentiation toward odontoblasts $(40,41)$. Furthermore, OCN and BSP are osteoblast-specific markers, and their promoters contain Runx2 binding sites $(38,39)$. Upregulating the expression of OCN and BSP was previously indicated to significantly promote the differentiation of MSCs into osteoblasts $(40,41)$. The present data indicates that the inhibition of the Rho/ROCK signaling pathway would not impact odontoblast differentiation in the early stage of odontoblast differentiation, but promote the differentiation in the late stage. This suggests that the inhibitor only functions in the late stage.

In conclusion, the present work indicated that the expression of Runx2 initially increases and then decreases during differentiation of hDPSCs into odontoblasts, whereas in hBMSCs, Runx 2 is consistently and highly expressed during osteoblast differentiation. Furthermore, the present findings suggested that inhibition of the RhoA/ROCK signaling pathway with $\mathrm{C} 3$ exoenzyme significantly downregulated the expression of Runx2, but had no significant impact on odontoblast differentiation in the early stage. However, differentiation in the late stage appeared to be promoted, suggesting that it may be possible to direct the differentiation of hDPSCs into odontoblasts by inhibiting the RhoA/ROCK signaling pathway to downregulate the expression of Runx2.

\section{References}

1. Corbella S, Taschieri S, Samaranayake L, Tsesis I, Nemcovsky C and Del Fabbro M: Implant treatment choice after extraction of a vertically fractured tooth. A proposal for a clinical classification of bony defects based on a systematic review of literature. Clin Oral Implants Res 25: 946-956, 2014.

2. Ortega-Martinez J, Pérez-Pascual T, Mareque-Bueno S, Hernández-Alfaro F and Ferrés-Padró E: Immediate implants following tooth extraction. A systematic review. Med Oral Patol Oral Cir Bucal 17: e251-e261, 2012.

3. Gonda T, Ikebe K, Ono T and Nokubi T: Effect of magnetic attachment with stress breaker on lateral stress to abutment tooth under overdenture. J Oral Rehabil 31: 1001-1006, 2004.

4. Zhao Y, Wang L, Jin Y and Shi S: Fas ligand regulates the immunomodulatory properties of dental pulp stem cells. J Dent Res 91: 948-854, 2012.

5. Ledesma-Martinez E, Mendoza-Núñez VM and Santiago-Osorio E: Mesenchymal stem cells derived from dental pulp: A review. Stem Cells Int 2016: 4709572, 2016.

6. Nuti N, Corallo C, Chan BM, Ferrari M and Gerami-Naini B: Multipotent differentiation of human dental pulp stem cells: A literature review. Stem Cell Rev 12: 511-523, 2016.

7. Kabir R, Gupta M, Aggarwal A, Sharma D, Sarin A and Kola MZ: Imperative role of dental pulp stem cells in regenerative therapies: A systematic review. Niger J Surg 20: 1-8, 2014.

8. Shi S, Robey PG and Gronthos S: Comparison of human dental pulp and bone marrow stromal stem cells by cDNA microarray analysis. Bone 29: 532-539, 2001.

9. Téclès O, Laurent P, Zygouritsas S, Burger AS, Camps J, Dejou J and About I: Activation of human dental pulp progenitor/stem cells in response to odontoblast injury. Arch Oral Biol 50: 103-108, 2005.

10. Wang J, Dong H, Wang W and Gu JD: Reverse-transcriptional gene expression of anammox and ammonia-oxidizing archaea and bacteria in soybean and rice paddy soils of Northeast China. Appl Microbiol Biotechnol 98: 2675-2686, 2014.

11. Nosrat IV, Smith CA, Mullally P, Olson L and Nosrat CA: Dental pulp cells provide neurotrophic support for dopaminergic neurons and differentiate into neurons in vitro; implications for tissue engineering and repair in the nervous system. Eur J Neurosci 19: 2388-2398, 2004.

12. Király M, Porcsalmy B, Pataki A, Kádár K, Jelitai M, Molnár B, Hermann P, Gera I, Grimm WD, Ganss B, et al: Simultaneous PKC and cAMP activation induces differentiation of human dental pulp stem cells into functionally active neurons. Neurochem Int 55: 323-332, 2009.

13. Zhang W, Walboomers XF, Shi S, Fan M and Jansen JA: Multilineage differentiation potential of stem cells derived from human dental pulp after cryopreservation. Tissue Eng 12: 2813-2823, 2006. 
14. Petrovic V and Stefanovic V: Dental tissue-new source for stem cells. ScientificWorldJournal 9: 1167-1177, 2009.

15. Sun SS, Zhang L, Yang J and Zhou X: Role of runt-related transcription factor 2 in signal network of tumors as an inter-mediator. Cancer Lett 361: 1-7, 2015.

16. Wojtowicz AM, Templeman KL, Hutmacher DW, Guldberg RE and Garcia AJ: Runx2 overexpression in bone marrow stroma cells accelerates bone formation in critical-sized femoral defects. Tissue Eng Part A 16: 2795-2808, 2010.

17. Livak KJ and Schmittgen TD: Analysis of relative gene expression data using real-time quantitative PCR and the 2(-Delta Delta C(T)) method. Methods 25: 402-408, 2001.

18. Ducy P, Zhang R, Geoffroy V, Ridall AL and Karsenty G: Osf2/Cbfa1: A transcriptional activator of osteoblast differentiation. Cell 89: 747-754, 1997.

19. Xu N, Guan L, He Y, Li D, Han B and Yang F: Dynamic expression of Runx2 during the differnetiation of odontoblsts and osteoblasts. Chin J Conser Dent 23: 209-13, 2013 (In Chinese).

20. Nasu K, Tsuno A, Yuge A and Narahara H: Mevalonate-Ras homology (rho)/rho-associated coiled-coil-forming protein kinase (ROCK)-mediated signaling pathway as a therapeutic target for the treatment of endometriosis-associated fibrosis. Curr Signal Transduct Ther 5: 141-148, 2010.

21. Niu LN, Sun JQ, Li QH, Jiao K, Shen LJ, Wu D, Tay F and Chen JH: Intrafibrillar-silicified collagen scaffolds enhance the osteogenic capacity of human dental pulp stem cells. J Dent 42: 839-849, 2014

22. Gronthos S, Arthur A, Bartold PM and Shi S: A method to isolate and culture expand human dental pulp stem cells. Methods Mol Biol 698: 107-121, 2011

23. Bruderer M, Richards RG, Alini M and Stoddart MJ: Role and regulation of RUNX2 in osteogenesis. Eur Cell Mater 28: 269-286, 2014

24. Gronthos S, Mankani M, Brahim J, Robey PG and Shi S: Postnatal human dental pulp stem cells (DPSCs) in vitro and in vivo. Proc Natl Acad Sci USA 97: 13625-13630, 2000

25. He WX, Niu ZY, Zhao SL, Jin WL, Gao J and Smith AJ: TGF-beta activated Smad signalling leads to a Smad3-mediated down-regulation of DSPP in an odontoblast cell line. Arch Oral Biol 49: 911-918, 2004.

26. Rocha ÉD, de Brito NJ, Dantas MM, Silva Ade A, Almeida Md and Brandão-Neto J: Effect of zinc supplementation on $\mathrm{GH}$, IGF1, IGFBP3, OCN, and ALP in non-zinc-deficient children. J Am Coll Nutr 34: 290-299, 2015.

27. Vimalraj S, Arumugam B, Miranda PJ and Selvamurugan N Runx2: Structure, function, and phosphorylation in osteoblast differentiation. Int J Biol Macromol 78: 202-208, 2015.

28. Feng Y, LoGrasso PV, Defert O and Li R: Rho kinase (ROCK) inhibitors and their therapeutic potential. J Med Chem 59: 2269-2300, 2016.

29. Julian L and Olson MF: Rho-associated coiled-coil containing kinases (ROCK): Structure, regulation, and functions. Small GTPases 5: e29846, 2014.
30. Tripathi BK, Grant T, Mertins P, Qian X, Wang D, Papageorge AG, Carr SA and Lowy DR: AKT positively regulates Rho-GTP by attenuating the GAP activity of the DLC1 tumor suppressor: A mechanistic study with translational implications. Cancer Res 76 (14 Suppl): S4377, 2016.

31. Menna L, Pablo and Cardama: PROTEINAS DE LIGACAO AO GTP [Descritor de assunto]. Front Psychol 6: 623-626, 2015.

32. Nour-Eldine W, Ghantous CM, Zibara K, et al. Adiponectin Attenuates Angiotensin II-Induced Vascular Smooth Muscle Cell Remodeling through Nitric Oxide and the RhoA/ROCK Pathway[J]. Frontiers in Pharmacology 7: 86, 2016.

33. Prowse PD, Elliott CG, Hutter J and Hamilton DW: Inhibition of Rac and ROCK signalling influence osteoblast adhesion, differentiation and mineralization on titanium topographies. PLoS One 8: e58898, 2013.

34. Tang Z, Shi D, Jia B, Chen J, Zong C, Shen D, Zheng Q, Wang J and Tong X: Exchange protein activated by cyclic adenosine monophosphate regulates the switch between adipogenesis and osteogenesis of human mesenchymal stem cells through increasing the activation of phosphatidylinositol 3-kinase. Int $\mathrm{J}$ Biochem Cell Biol 44: 1106-1120, 2012.

35. Santos A, Bakker AD, de Blieck-Hogervorst JM and Klein-Nulend J: WNT5A induces osteogenic differentiation of human adipose stem cells via rho-associated kinase ROCK. Cytotherapy 12: 924-932, 2010.

36. Kusuyama J, Bandow K, Shamoto M, Kakimoto K, Ohnishi T and Matsuguchi T: Low intensity pulsed ultrasound (LIPUS) influences the multilineage differentiation of mesenchymal stem and progenitor cell lines through ROCK-Cot/Tpl2-MEK-ERK signaling pathway. J Biol Chem 289: 10330-10344, 2014.

37. Li G, Liu L, Shan C, Cheng Q, Budhraja A, Zhou T, Cui H and Gao N: RhoA/ROCK/PTEN signaling is involved in AT-101-mediated apoptosis in human leukemia cells in vitro and in vivo. Cell Death Dis 5: e998, 2014.

38. Chen S, Gluhak-Heinrich J, Wang YH, Wu YM, Chuang HH, Chen L, Yuan GH, Dong J, Gay I and MacDougall M: Runx2, osx, and dspp in tooth development. J Dent Res 88: 904-909, 2009.

39. Li S, Kong H, Yao N, Yu Q, Wang P, Lin Y, Wang J, Kuang R, Zhao $\mathrm{X}$ and $\mathrm{Xu} \mathrm{J}$ : The role of runt-related transcription factor 2 (Runx2) in the late stage of odontoblast differentiation and dentin formation. Biochem Biophys Res Commun 410: 698-704, 2011.

40. Gao RT, Zhan LP, Meng C, Zhang N, Chang SM, Yao R and Li C: Homeobox B7 promotes the osteogenic differentiation potential of mesenchymal stem cells by activating RUNX2 and transcript of BSP. Int J Clin Exp Med 8: 10459-10470, 2015.

41. Zhang X, Ma Y, Fu X, Liu Q, Shao Z, Dai L, Pi Y, Hu X, Zhang J, Duan $\mathrm{X}$, et al: Runx2-modified adipose-derived stem cells promote tendon graft integration in anterior cruciate ligament reconstruction. Sci Rep 6: 19073, 2016. 